\title{
Les facteurs d'apparition du syndicalisme catholique dans l’imprimerie et les déterminants de la stratégie syndicale 1921-1945
}

\section{Luc Desrochers}

Volume 37, numéro 2, septembre 1983

Travailleurs et mouvements sociaux

URI : https://id.erudit.org/iderudit/304156ar

DOI : https://doi.org/10.7202/304156ar

Aller au sommaire du numéro

Éditeur(s)

Institut d'histoire de l'Amérique française

ISSN

0035-2357 (imprimé)

1492-1383 (numérique)

Découvrir la revue

Citer cet article

Desrochers, L. (1983). Les facteurs d'apparition du syndicalisme catholique dans l'imprimerie et les déterminants de la stratégie syndicale 1921-1945. Revue d'histoire de l'Amérique française, 37(2), 241-269.

https://doi.org/10.7202/304156ar d'utilisation que vous pouvez consulter en ligne. 


\title{
LES FACTEURS D'APPARITION DU SYNDICALISME CATHOLIQUE DANS L'IMPRIMERIE ET LES DETERMINANTS DE LA STRATÉGIE SYNDICALE 1921-1945
}

\author{
LUC DESROCHERS \\ Département d'Histoire \\ Université du Québec à Montréal
}

Quand on se penche sur le syndicalisme dans l'industrie canadienne de l'imprimerie, il ressort que les unions internationales ont possédé presque un monopole de représentation syndicale depuis les années 1860-1870. À l'origine, l'Union typographique internationale exerçait seule ce monopole. Au tournant du siècle toutefois, les innovations technologiques ont provoqué une telle différenciation dans les activités qui composaient alors le métier d'imprimeur que plusieurs de ces activités sont devenues des métiers spécifiques ${ }^{1}$. L'un des effets de ces transformations de l'industrie fut la formation de nouvelles unions internationales d'imprimeurs créées à partir de l'Union typographique internationale, en marge d'elle ou par l'action conjointe d'affiliées de l'UTI et d'unions indépendantes. Ainsi, entre 1889 et 1915, cinq de ces unions ont été créées par les pressiers, les relieurs, les lithographes, etc.

Cet accroissement du nombre des unions internationales d'imprimeurs et l'augmentation de leur monopole de représentation dans l'industrie n'ont pas empêché la manifestation de volontés autonomistes parmi les syndiqués canadiens de l'imprimerie. Bien au contraire, c'est justement cette quasi-omniprésence des unions internationales et surtout son caractère centralisateur qui ont engendré différentes tentatives de formation d'une fédération canadienne de travailleurs-imprimeurs ${ }^{2}$. L'une de ces tentatives, la plus durable, va s'inscrire dans le développement du mouvement syndical catholique au Québec. Ce sont les conditions qui ont entouré l'implantation de ce type de syndicalisme chez les imprimeurs québécois que nous nous proposons de présenter dans

Pour ce qui est des conséquences des changements technologiques sur la structure syndicale dans l'industrie de l'imprimerie, on consultera avec grand profit les ouvrages de Elizabeth F. Baker, Displacement of Men by Machines (N.Y., Columbia University Press, 1933), 284 p. et Printers and Technology (N.Y., Greenwood Press, 1974), 545 p. Voir aussi A. Zimbalist, «Technology and the Labor Process in the Printing Industry,» dans A. Zimbalist, Cases Studies on the Labor Process (N.Y. and London, Monthly Review Press, 1979): 103-126.

Voir E. Forsey, Trade Unions in Canada, 1812-1902 (Toronto, UTP, 1982), 107, 111, 207-208, 214, 219, 224, 379. Voir aussi R. Babcock, Gompers in Canada (Toronto, UTP, 1974), $152-153$.

RHAF, vol. 37, no 2, septembre 1983 
les pages qui suivent. Nous essaierons également de mettre en évidence les facteurs qui ont conditionné la stratégie et la pratique des syndicats catholiques de l'imprimerie durant leur 24 premières années d'existence.

$$
* *
$$

\section{Le mouvement des 44 heures}

L'arrivée de ces nouveaux venus que sont les syndicats catholiques d'imprimeurs s'inscrit dans un contexte très particulier du point de vue économique et syndical. Comme toute l'économie canadienne, l'industrie de l'imprimerie est marquée par une récession qui s'étend de 1921 à 1924. Au Québec, le malaise se perpétue même jusqu'en 1926 en ce qui concerne certains secteurs de l'industrie. (Tableau I). Les données présentées au tableau ci-dessus montrent en effet que la valeur de la production de l'impression-édition n'augmente que de $8.6 \%$ de 1922 à 1925, alors que durant la période 1919-1922 son taux d'accroissement avait été de $40.5 \%$. Dans l'impression-reliure, cette même valeur ne progresse que de $0.4 \%$ entre 1924 et 1926 . Conséquemment à cette situation difficile, l'impression-édition connaît une baisse importante du nombre d'établissements, de 74 en 1922 à 55 en 1925, soit $25.7 \%$. La main-d'oeuvre qui y est employée stagne de 1924 à 1926. D'autre part, malgré un accroissement remarquable de $24.5 \%$ du nombre d'ateliers de 1923 à 1924, l'emploi dans l'impression-reliure demeure sensiblement au même niveau durant les années 1924 à 1926.

Comme cela se produit généralement, la situation de crise a des conséquences immédiates sur la force des organisations syndicales. Les effectifs canadiens de l'Union typographique internationale, par exemple, passent de 5100 en 1921 à 4200 en $1924^{3}$, soit une diminution de $17.6 \%$. À Montréal, la diminution des effectifs de l'ensemble des unions internationales est encore plus dramatique puisqu'elles perdent $24.5 \%$ de leurs membres de 1921 à 1929. (Voir Tableau II)

Par contre, la fondation par les imprimeurs québécois de trois syndicats catholiques en deux mois durant 1921 , année où débute la récession, a de quoi surprendre. Pour expliquer ce phénomène d'autres facteurs que la conjoncture économique entrent en ligne de compte.

En effet, en 1921, les internationaux ont lancé un mouvement de grève qui a joué un rôle important dans la création des syndicats catholiques de l'imprimerie. Les représentants du Syndicat catholique des typographes de Montréal en parleront plus tard en ces termes:

\footnotetext{
3 H.A. Logan, Trade Unions in Canada, Their Development and Functioning (Toronto, Macmillan, 1948), 617.
} 


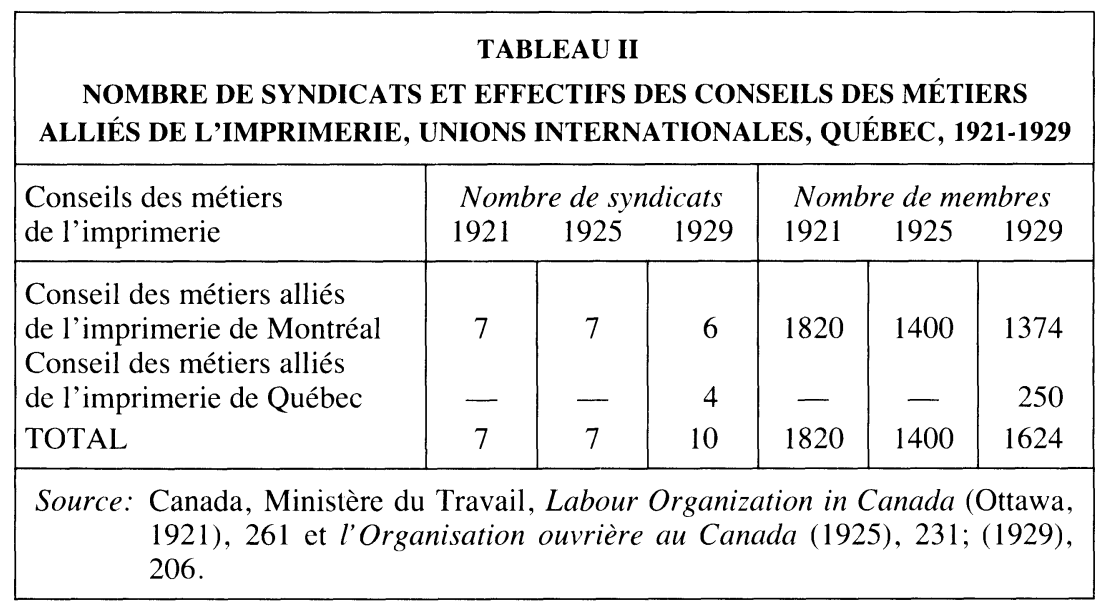

$\mathrm{Au} 1^{\mathrm{er}}$ juillet, c'est-à-dire en plein début de morte-saison, des mages venus du Méridion avaient déclenché chez-nous une grève bizarre qui avait sinon complètement désorganisé le métier, du moins créé un état de choses qui était une belle imitation de marasme. ${ }^{4}$

Malgré ce qu'en disent les typographes catholiques, la grève n'est évidemment pas la cause de la crise qui affecte l'industrie de l'imprimerie à ce moment. D'autre part, le mouvement de grève dont ils parlent n'a pas débuté le premier jour de juillet mais bien deux mois auparavant. Le $1^{\mathrm{er}}$ mai de cette année avait été choisi comme date d'implantation de la semaine de travail de 44 heures dans toute l'industrie de l'imprimerie commerciale nord-américaine. C'est l'UTI qui avait fixé cet objectif et qui avait planifié le mouvement de grève pour l'atteindre ${ }^{5}$. Dans le cadre du Mouvement des 44 heures (c'est le nom qu'on lui a donné), les imprimeurs du Canada ont mené une lutte qui a dépassé en durée et en coûts financiers toutes celles faites dans le même but par les unités américaines affiliées à l'UTI; ce qui a suscité énormément de mécontentement chez les syndiqués canadiens ${ }^{6}$.

Toujours est-il que les imprimeurs du Canada lancent leur mouvement de grève le 2 mai 1921 . Le mot d'ordre est suivi de telle sorte que durant le mois de juillet suivant dix grèves sont en marche, ce qui fait d'eux le plus important groupe de grévistes au Canada ${ }^{7}$. En fait, les

\footnotetext{
4 Album-Souvenir, 15 ${ }^{\mathrm{e}}$ anniversaire de la FCMIC, 1925-1940, 21 (désormais Album-Souvenir...)

5 Voir E.F. Baker, Printers..., 305.

6 Voir à ce propos Sally F. Zerker, The Rise and Fall of the Toronto Typographical Union, 1832-1972 (Toronto, UTP, 1982), chap. 9: 178 à 204.

7 Ministère du Travail, La Gazette du Travail, Ottawa, août 1921, 1078.
} 


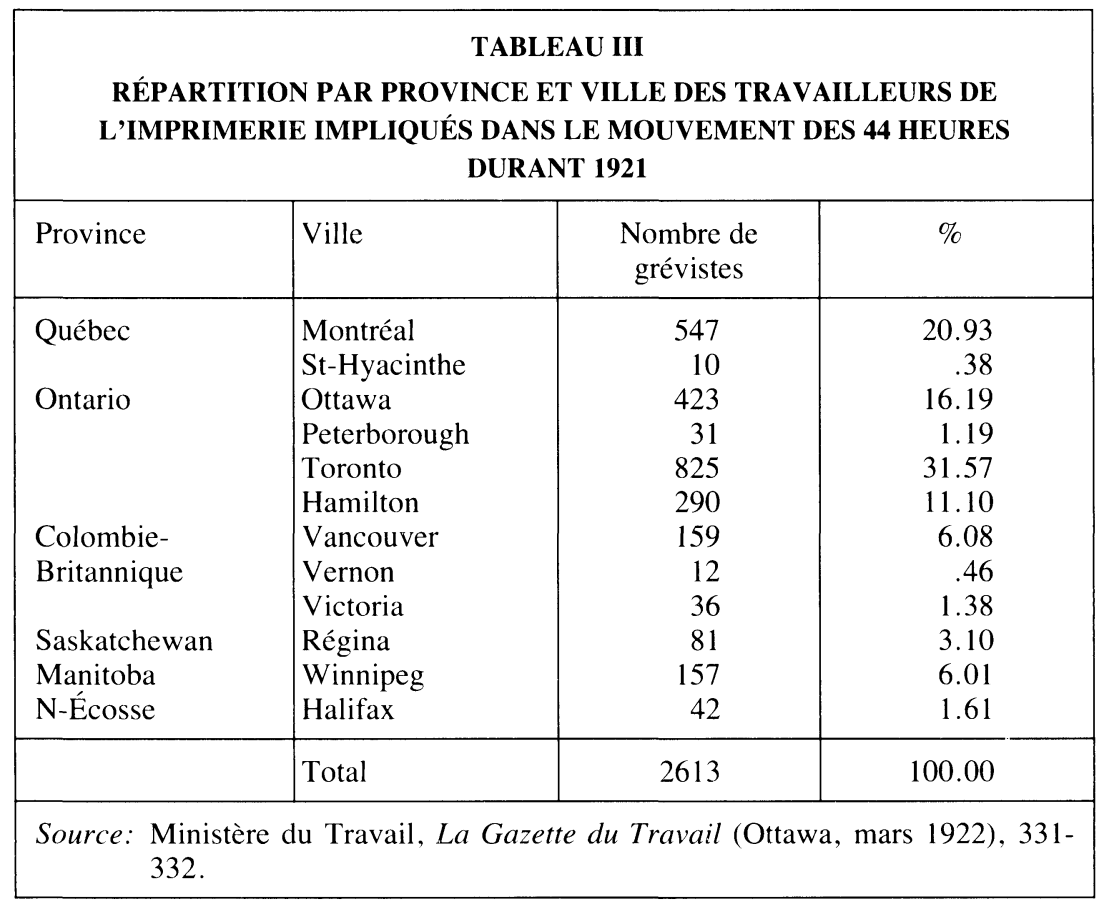

effectifs unionistes canadiens engagés dans le Mouvement des 44 heures au cours de l'année se sont répartis comme suit:

Selon toute vraisemblance, $50 \%$ des effectifs canadiens de l'Union typographique internationale sont en grève à ce moment. Malheureusement, la grève traîne en longueur provoquant par le fait même un effritement des effectifs grévistes et une augmentation de l'importance relative des grévistes canadiens qui y sont impliqués. S. Zerker, dans son étude sur l'Union typographique de Toronto, l'a d'ailleurs mesurée précisément. En avril 1923, parmi les 3523 grévistes appartenant à l'Union typographique internationale, 2127 sont canadiens. Ils représentent donc $60 \%$ des typographes impliqués dans le conflit. En 1924, les locaux de Montréal, Ottawa, Toronto et Hamilton seront toujours en grève ${ }^{8}$.

En ce qui a trait à la participation des syndiqués du Québec, nous pouvons dire qu'elle est plus que significative. Comme le tableau IV

8 S.F. Zerker, op. cit., 396. Faisons remarquer que l'auteur mentionne une grève à Québec en 1924 mais qu'aucun conflit n'est recensé pour cette ville durant cette période dans La Gazette du Travail, fév. 1925, 150-151. Le seul conflit recensé durant les années qui nous intéressent est celui de février 1922. La traduction est de nous. 
l'indique, 485 et 530 d'entre eux sont en grève en 1921 et 1922 respectivement. Mais dès l'année suivante leurs rangs s'éclaircissent énormément. À Montréal, trois grèves d'imprimeurs se termineront à la date décrétée par l'UTI comme ultime limite de toute grève pour l'obtention de la semaine de travail de 44 heures, c'est-à-dire le 30 août $1924^{9}$. Le mouvement aura finalement duré trois années durant lesquelles les grévistes ont vu leurs rangs se clairsemer, tout comme les effectifs de l'UTI d'ailleurs.

Il n'a pas été nécessaire d'attendre le dénouement du conflit pour que des signes évidents de mécontentement apparaissent parmi les syndiqués de l'imprimerie du Québec. L'attitude intransigeante de l'UTI quant au déclenchement et à l'application du programme du Mouvement des 44 heures jointe à la crise économique qui affectait déjà l'industrie firent en sorte que les doléances à propos du droit au chapitre des syndicats canadiens dans l'orientation des unions internationales trouvèrent une occasion propice de se manifester à nouveau. Car, effectivement, ces récriminations ne sont pas nouvelles de la part des syndiqués canadiens. Les membres des syndicats de Montréal avaient déjà demandé à plusieurs reprises une représentation spécifiquement canadienne au Conseil exécutif de cette Internationale. S. Zerker a relevé quatre résolutions en ce sens entre 1916 et 1921, la dernière ayant eu lieu le 6 août 1921, c'est-à-dire à peine un mois après que le mouvement de grève eut atteint Montréal ${ }^{10}$. Mais à la différence des années précédentes, le mécontentement suscité par le Mouvement des 44 heures ne provoquera pas que des récriminations, il aura aussi des conséquences auxquelles les internationaux ne s'attendaient sûrement pas.

\section{L'apparition des syndicats catholiques d'imprimeurs}

Comme nous allons le voir, c'est dans ce contexte économique et syndical tout à fait particulier que va se développer le syndicalisme catholique dans l'imprimerie. Il existait bien sûr des imprimeurs appartenant à des syndicats catholiques avant cette époque. Ainsi, la Corporation ouvrière catholique de Trois-Rivières a eu une section composé d'imprimeurs jusqu'en $1917^{11}$ et un syndicat catholique d'imprimeurs a vu le jour à Québec en 1916. C'est à la suite d'une grève menée par l'Union internationale à laquelle appartenaient les typographes de l'Action catholique que celui-ci fut fondé ${ }^{12}$. À notre connaissance, ces typographes ont fondé le premier syndicat composé exclusivement

\footnotetext{
9 Cette échéance avait été fixée en juillet de la même année. Voir Ministère du Travail, $L a$ Gazette du Travail, Ottawa, août 1924, 699.

10 S.F. Zerker, op. cit., 204 et note 152, 371.

11 Ministère du Travail, Labor Organization in Canada (Ottawa, 1917), 221. Sur la Corporation voir J. Rouillard, Les Syndicats nationaux au Québec de 1900 à 1930 (Québec, PUL, 1979), 191 à 193.

12 Programme-Souvenir..., (1944): 9.
} 


\begin{tabular}{|c|c|c|c|c|c|c|c|c|c|c|c|c|c|c|}
\hline \multicolumn{15}{|c|}{$\begin{array}{c}\text { TABLEAU IV } \\
\text { ÉVOLUTION DE LA PARTICIPATION DES TYPOGRAPHES QUÉBÉCOIS AU MOUVEMENT DES } 44 \text { HEURES, 1921-1924 }\end{array}$} \\
\hline \multirow{3}{*}{$\begin{array}{l}\text { OCCUPATION } \\
\text { Imprimeurs }\end{array}$} & \multirow{3}{*}{\begin{tabular}{|l} 
VILLE \\
St- \\
Hyacinthe
\end{tabular}} & \multicolumn{8}{|c|}{ NB. IMPLIQUE } & \multirow{2}{*}{\multicolumn{4}{|c|}{$\begin{array}{l}\text { JOURS OUVRABLES } \\
\text { PERDUS }\end{array}$}} & \multirow{3}{*}{$\begin{array}{c}\text { DURÉE } \\
\text { EN JRS. OUV } \\
\begin{array}{c}\text { TOTAL } \\
16\end{array}\end{array}$} \\
\hline & & \multicolumn{4}{|c|}{ PATRONS } & \multicolumn{4}{|c|}{ EMPLOYÉS } & & & & & \\
\hline & & $\begin{array}{c}1921 \\
2\end{array}$ & 1922 & 1923 & 1924 & $\begin{array}{c}1921 \\
10\end{array}$ & 1922 & 1923 & 1924 & $\begin{array}{c}1921 \\
160\end{array}$ & 1922 & 1923 & 1924 & \\
\hline Compositeurs & Mtl. & 1 & 1 & 1 & 1 & 25 & 25 & 10 & 8 & 4,225 & 7,081 & 3,070 & 1,120 & 987 \\
\hline $\begin{array}{l}\text { Imprimeurs- } \\
\text { Compositeurs }\end{array}$ & Mtl. & 55 & 40 & 9 & - & 450 & 370 & 140 & 117 & 63,140 & 106,215 & 42,980 & 18,095 & 972 \\
\hline Compositeurs & $\begin{array}{l}\text { Garden- } \\
\text { vale }\end{array}$ & & 1 & & & & 10 & & & & 210 & & & 21 \\
\hline Imprimeurs & Québec & & 4 & & & & 100 & & & & 1,600 & & & 16 \\
\hline Compositeurs & Mtl. & & 1 & 1 & 1 & & 25 & 12 & 12 & & 2,275 & 3,684 & 2,448 & 602 \\
\hline
\end{tabular}

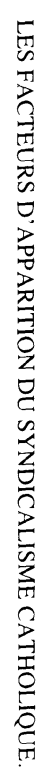


d'imprimeurs ${ }^{13}$, mais son influence n'a pas dépassé les frontières de la capitale provinciale. Il n'a pas non plus joué de rôle important dans la création de la Fédération catholique des métiers de l'imprimerie du Canada (FCMIC) en $1925^{14}$. Cette initiative revient plutôt aux syndicats catholiques d'imprimeurs de Montréal et de Hull.

Le 4 août 1921, le syndicalisme catholique faisait en effet son entrée dans l'industrie de l'imprimerie de Montréal en tant qu'alternative à l'unionisme international sous la forme du Syndicat catholique et national des typographes de Montréal ${ }^{15}$. Ce sont donc, vraisemblablement, une partie de ceux qui ressentaient le plus durement le manque d'influence des unions canadiennes sur les orientations de l'UTI qui ont «ouvert le bal». La mise sur pied du Syndicat catholique des typographes a immédiatement eu un effet d'entraînement car lors de sa seconde assemblée, le 23 août, donc trois semaines après sa fondation, un autre syndicat catholique vit le jour: le Syndicat catholique et national des pressiers. Cette nouvelle unité syndicale fut formée par quatorze pressiers de Montréal dont une minorité provenait du secteur journal tandis que les autres étaient issus du secteur commercial ${ }^{16}$.

Après Montréal, c'est à la région de l'Outaouais que le syndicalisme catholique a étendu ses ramifications. Au cours de septembre, les efforts de deux employés du journal Le Droit de Hull, propriété des Oblats, aboutirent à la formation d'un syndicat catholique d'imprimeurs. S'il faut en croire certains documents syndicaux, le Syndicat catholique des imprimeurs-relieurs du diocèse d'Ottawa aurait été formé à la demande expresse du Père J. Bonhomme, o.m.i., aumônier de l'Association ouvrière catholique de Hull $(\mathrm{AOCH})^{17}$. Mais à y regarder de plus près, on s'aperçoit que l'explication n'est pas si simple. La thèse avancée par $M$. Têtu à ce sujet veut que les travailleurs des ateliers de ce journal aient quitté «l'internationale» suite à leur décision de ne pas participer à une grève de solidarité; il attribue cette désaffiliation à l'intervention du père Bonhomme et d'un de ses confrères ${ }^{18}$. D'autre part, des informations rapportées par B. Hogan viennent préciser la dynamique qui a pu exister entre le mouvement de grève des internationaux et l'action des Oblats, celle du père Bonhomme en particulier. En effet, l'auteur tire les renseignements suivants d'un document d'époque daté du 1 er juin 1921: «Les ouvrières hydrographes [il s'agit ici, selon toute vraisemblance, des ouvriers typographes] à l'atelier du (Droit)

\footnotetext{
13 Nous ignorons la date de fondation de la section des imprimeurs de la Corporation ouvrière catholique de Trois-Rivières.

${ }_{14}$ CTCC, Procès-verbal du Congrès, 18 sept. 1926, 65.

15 Programme-Souvenir..., (1943): 47; Album-Souvenir..., 21 et L'Imprimeur, mai-juin 1950,40 .

16 Album-Souvenir..., 24, 37; L'Imprimeur, mai-juin 1950, 17

17 Album-Souvenir..., 53; L'Imprimeur, mai-juin 1950, 10.

18 M.Têtu, Les premiers syndicats catholiques canadiens, 1900-1921 (thèse de Doctoratès-Lettres, U. Laval, 1962), 136.
} 
viennent de déclarer la grève. Ils demandent 44 hrs (sic) de travail au lieu de 48 et $10 \$$ d'augmentation par semaine.» ${ }^{19}$

La concordance entre le moment du débrayage et les débuts du Mouvement des 44 heures, de même que l'identité des revendications ne laissent aucun doute à notre avis: l'union des typographes affiliée à l'UTI et regroupant les employés de ce métier à l'emploi du journal $L e$ Droit a bel et bien participé, bon gré mal gré, au Mouvement des 44 heures. Ce qui n'a pas semblé être très apprécié par la direction du journal puisque le document cité par Hogan mentionne que «Le Droit préfère fermer ses portes [plutôt] que de se plier à des conditions aussi excessives ${ }^{20}$. Au lieu d'attendre d'en être réduite à cette extrémité, la direction entrevoit une autre solution: créer un syndicat catholique d'imprimeurs, ce qui est fait en septembre. Pour s'assurer du bon fonctionnement du nouveau syndicat, on le dotera d'un aumônier, le père Bonhomme évidemment, ainsi que d'un agent d'affaires des plus expérimentés et convaincu de la cause syndicale catholique, Achille Morin, l'un des fondateurs de l'AOCH en $1912^{21}$.

La percée du syndicalisme confessionnel dans l'industrie de l'imprimerie, fief presqu'exclusivement réservé aux unions internationales, et le moment où elle se produisait n'avaient rien de rassurant pour les dirigeants internationaux. D'autant plus que, dans le cas des typographes montréalais tout au moins, aucun indice ne nous permet de croire que l'abandon des rangs des unions internationales par certains de leurs membres soit dû à une quelconque intervention cléricale. Le silence des membres du Syndicat catholique des typographes de Montréal est très significatif à cet égard. Il nous laisse croire que d'autres motifs sont à l'origine de la fondation de leur syndicat. Bien plus, la référence continuelle à la grève de 1921 nous permet d'affirmer que c'est là que réside le facteur essentiel de sa création. Aux dires mêmes du secrétaire du syndicat de 1940:

Ce fut une des causes qui influencèrent le plus la fondation du Syndicat en en faisant pour ainsi dire toucher du doigt l'urgente nécessité. Le malaise créé par cette grève inopportune - tournant au <lock-out dans bien des cas - eut pour effet de diriger nombre d'ouvriers vers un mouvement syndical autonome, où seuls des

19 B. Hogan, «Church and Union: The Case of Hull», dans Labour/Le Travailleur, \#7, printemps 1981, 146, note 5. Le document cité est le Codex Historicus (1911-1925) trouvé en août 1980 et maintenant déposé dans les Archives Deschâtelets des Oblats de Hull.

20 Ibidem.

21 Idem, 141. 
gens de chez-nous, ayant égard aux circonstances de chez-nous, pourraient faire chez-nous la pluie ou le beau temps. ${ }^{22}$

Cette explication est crédible si on se rappelle que ce sont les unions de typographes montréalais qui revendiquaient une représentation canadienne à la direction de l'UTI. Si l'insatisfaction à l'égard des réponses apportées à ces demandes avait l'importance que nous croyons, il n'y a rien de surprenant à ce que ce soit à Montréal que soient apparus les syndicats catholiques d'imprimeurs destinés à donner à ce type de syndicalisme l'allure d'un véritable mouvement.

Pour ce qui est de déterminer qui, du clergé, des dirigeants laïques du mouvement syndical catholique ou des pressiers eux-mêmes a eu l'initiative de former un syndicat catholique des pressiers, on ne peut que faire des hypothèses. Par contre, le cas du syndicat de Hull est beaucoup plus probant. Il montre avec évidence que c'est la coïncidence du Mouvement des 44 heures et de l'action cléricale qui va permettre la création de ce syndicat. Le mouvement de grève apparaît même comme indispensable à la réalisation de l'objectif clérical car la volonté de former une unité syndicale catholique d'imprimeurs devait dater de quelques années déjà. La preuve en est que Armand Morin et Louis-Philippe Riel, les deux employés du Droit «à l'origine» du syndicat, avaient collaboré à la fondation du Cercle d'études Benoît XV de Hull en $1918^{23}$, un organisme voué à la diffusion de la doctrine sociale de l'Église chez les travailleurs et à la formation de dirigeants syndicaux catholiques.

De tous les syndicats catholiques d'imprimeurs qui furent mis sur pied durant le Mouvement des 44 heures, un seul ne doit pas sa création à un quelconque effet du mouvement de grève des internationaux. Il s'agit du Syndicat catholique et national des pressiers de journaux (no 2) qui est né d'une division à l'intérieur des rangs du syndicat catholique de pressiers formé en août 1921. Les pressiers de journaux estimaient que leur réunion avec les pressiers de l'imprimerie commerciale leur posait certaines difficultés. Ils alléguaient qu'étant trop peu nombreux, ils ne pouvaient prendre une part utile aux délibérations des assemblées ${ }^{24}$. C'est pourquoi il fut convenu de former un groupe exclusif de pressiers de journaux ayant sa propre constitution et sa propre direction.

C'est ainsi qu'en octobre 1922, un nouveau syndicat catholique d'imprimeurs venait s'ajouter aux quatre déjà existants.

22 Album-Souvenir..., 21. Les soulignés sont du secrétaire du syndicat. Voir aussi la p. 7 où on reprend la même argumentation mais à propos de l'avènement de la Fédération catholique des imprimeurs en 1925. Bien sûr, on peut souligner que cette citation est postérieure de 19 ans aux événements en cause et qu'à ce titre elle doit être utilisée sous toutes réserves. Nous en convenons. Il n'en reste pas moins que dans les documents que nous avons consultés, cette explication prend toutes les allures d'un leitmotiv. Voir Programme-Souvenir..., (1943): 47 et L'Imprimeur, mai-juin 1950, 40.

23 Album-Souvenir..., 53.

24 Idem, 37. 
Il faudra attendre trois ans avant que d'autres syndicats ou associations de syndicats catholiques ne soient créés dans l'imprimerie. Ce qui nous permet d'affirmer que c'est à partir du Mouvement des 44 heures que l'on peut parler de l'implantation d'un mouvement en faveur du syndicalisme catholique chez les imprimeurs. Un mouvement pour lequel l'intervention cléricale constitue un facteur important, certes, mais insuffisant pour l'explication pleine et entière de son apparition. Pour que le désir de fonder des syndicats catholiques d'imprimeurs devienne une réalité, il fallait plus que la diffusion d'une doctrine, fusse-t-elle catholique, il fallait une occasion propice. Le Mouvement des 44 heures fut cette occasion.

\section{Pourquoi des syndicats catholiques?}

Le mécontentement à propos de la gouverne des unions internationales et le désir de posséder des syndicats autonomes, en plus de l'influence cléricale, ne suffisent pas à expliquer que certains imprimeurs du Québec se soient tournés vers le syndicalisme confessionnel plutôt que vers un syndicalisme national ${ }^{25}$ non-confessionnel. Pour comprendre ce choix des imprimeurs, il faut garder à l'esprit que l'établissement de syndicats catholiques au début des années 1920, dans l'imprimerie comme ailleurs, coïncide avec l'élimination graduelle des autres forces syndicales nationales au Québec. De 1906 à 1926, le nombre de syndicats nationaux au Québec ne cesse de diminuer, tandis que les syndicats catholiques, eux, connaissent une progression très importante (voir le Tableau V). Par exemple, en 1903, 90\% des 7000 membres que compte le Congrès national des métiers et du travail du Canada, devenu par la suite la Fédération canadienne du travail, sont québécois. En 1920, cette centrale canadienne compte encore 6000 membres mais seulement $10 \%$ d'entre eux sont du Québec. Avec la quasi-disparition de la FCT du Québec, les possibilités d'affiliation à une centrale syndicale autre qu'internationale sont réduites d'autant. D'autre part, l'opportunité de se retrouver parmi des gens de même culture et de même appartenance linguistique a certainement favorisé l'adhésion au syndicalisme catholique ${ }^{26}$.

À ces raisons s'ajoute le fait que le clergé catholique est directement impliqué dans l'industrie de l'imprimerie québécoise en tant que propriétaire d'ateliers ou en tant que client important. Du côté des syndiqués, on est donc en droit d'espérer un appui non équivoque de sa part, compte tenu qu'il est lui-même promoteur du syndicalisme catholique. De fait le clergé possède quelques journaux comme Le Droit de Hull et L'Action catholique de Québec auxquels il faut ajouter un

\footnotetext{
${ }^{25}$ Nous reprenons à notre compte le sens que $\mathrm{J}$. Rouillard a donné à cette expression dans Les Syndicats..., 9.

26 Idem, 120-121.
} 


\begin{tabular}{|c|c|c|c|c|}
\hline \multicolumn{5}{|c|}{$\begin{array}{c}\text { TABLEAU V } \\
\text { RÉPARTITION DES UNITÉS SYNDICALES PAR ALLÉGEANCE AU QUÉBEC } \\
1901-1931\end{array}$} \\
\hline Année & Internationaux & Nationaux & Catholiques & Total \\
\hline 1901 & 74 & 62 & & 136 \\
\hline 1906 & 155 & 81 & & 236 \\
\hline 1911 & 190 & & & 190 \\
\hline 1916 & 236 & 70 & 23 & 329 \\
\hline 1921 & 334 & 38 & 120 & 492 \\
\hline 1926 & 314 & 33 & 103 & 450 \\
\hline 1931 & 286 & 84 & 121 & 491 \\
\hline
\end{tabular}

ensemble de publications de tous genres faisant partie de ce qu'on appelait la «bonne presse» ${ }^{27}$. Il possède également quelques ateliers d'imprimerie, comme nous le verrons plus bas, mais c'est surtout en tant que consommateur de produits imprimés qu'il est susceptible de pouvoir favoriser l'implantation de syndicats catholiques d'imprimeurs ${ }^{28}$. En 1926, l'agent d'affaires des syndicats catholiques de l'imprimerie évaluait à $7 \$$ millions les travaux d'imprimerie faits chaque année à l'intention du clergé québécois ${ }^{29}$. Au moment de cette estimation, la valeur brute de la production dans l'édition et la reliure du Québec s'élevait à environ $22 \$$ millions $^{30}$. C'est dire, si l'évaluation de l'agent d'affaires est exacte, que $31.8 \%$ de la valeur de cette production était le résultat de commandes en provenance du clergé! C'est amplement suffisant pour conférer à quelque client que ce soit le pouvoir d'influencer plus d'un entrepreneur. C'est amplement suffisant aussi pour inciter des travailleurs de l'imprimerie en quête d'une structure syndicale qui leur soit propre à adhérer à ce mouvement syndical. Les résultats seront toutefois bien inférieurs aux possibilités de développement que semblait offrir cette adhésion.

Cinq ans après l'apparition des syndicats catholiques d'imprimeurs de Montréal et de Hull, seulement $75000 \$$ de produits imprimés

\footnotetext{
27 N.F. Ryan, The Clergy and Economic Growth in Quebec, 1896-1914 (Québec, PUL, 1966), 181-182.

${ }_{28}$ C'est ce qu'affirme H.A. Logan, op. cit., 574. Nos informations tendent par ailleurs à confirmer cette assertion.

29 Minutes des réunions du Syndicat catholique et national des relieurs de Montréal (désormais Minutes...), 13 oct. 1926, 19.

30 Ministère de l'Industrie et du Commerce, La province de Québec et ses manufactures, no 4 (Bois-Papier-Imprimerie, Québec, 1952), 91 et 96.
} 
étaient réalisés dans les ateliers ayant un syndicat catholique ${ }^{31}$, c'est-àdire moins de $2 \%$ de la valeur totale de la production destinée au clergé. De plus, seulement cinq syndicats catholiques seront formés de 1926 à 1934. Parmi ces syndicats mis sur pied en 1926 et 1927 essentiellement, ceux de Sherbrooke et de Joliette ne vivront qu'une année ${ }^{32}$.

Pour ce qui est des trois syndicats restants, celui de St-Hyacinthe a des origines qui nous sont complètement inconnues tandis que l'étude détaillée des minutes des assemblées du syndicat de Chicoutimi de 1926 à 1944 nous permet d'affirmer qu'aucune intervention cléricale n'a provoqué ou favorisé la création de ce syndicat. Il faut reconnaître toutefois que l'adhésion à une union internationale aurait été ni plus ni moins qu'un événement extraordinaire dans cette région du Saguenay-Lac-StJean où seuls existaient des syndicats catholiques.

Finalement, le seul syndicat qui doit sa création à l'action directe du clergé est celui que l'Imprimerie Beauchemin a imposé à ses employés à la demande d'un de ses clients priviligiés, le clergé bien sûr ${ }^{33}$. On ne peut donc pas présenter l'augmentation du nombre d'unités syndicales catholiques de l'imprimerie durant la décennie 1921-1931 comme la manifestation concrète du seul appui clérical au syndicalisme catholique dans cette industrie ${ }^{34}$, sinon avec force nuances. Ajoutons que s'en tenir uniquement au nombre de syndicats peut facilement induire en erreur puisque la formule syndicale en vigueur à cette époque est celle du syndicat de métier et qu'en conséquence chacun des nouveaux ateliers syndiqués ne donne pas nécessairement lieu à la formation d'un nouveau syndicat mais intègre plutôt ses travailleurs aux syndicats déjà existants en fonction des métiers qu'ils regroupent respectivement.

De toutes façons, le recours à l'influence du clergé en vue de l'accroissement des effectifs syndicaux et du nombre d'ateliers d'imprimerie sous contrôle catholique n'a pas donné non plus les résultats espérés. Par exemple, parmi les quinze ateliers ayant un contrat d'atelier fermé avec l'un ou l'autre des syndicats catholiques d'imprimeurs de Montréal, deux seulement appartiennent au clergé en 1928: celui de l'Institut des Sourds-Muets des Clercs de St-Viateur et celui de l'Imprimerie du Messager appartenant aux Jésuites ${ }^{35}$. On ne peut pas dire que les ateliers dirigés par le clergé soient les lieux de prédilection pour l'implantation de syndicats catholiques. D'autre part, au plan de l'évo-

\footnotetext{
31 Minutes..., 13 oct. $1926,19$.

32 Voir Ministère du Travail, L'Organisation ouvrière au Canada (Ottawa, 1927), 290 et Programme-Souvenir..., 1927: 29.

33 Voir J. Rouillard, Les Syndicats..., 238-239.

34 Idem, 234 à 240 notamment.

35 Pour la liste complète de ces ateliers voir Programme-Souvenir..., 1928: 31. Nous devons ajouter que dès 1926, ces syndicats affirment détenir trente contrats d'atelier fermé avec autant d'entreprises. Cependant, dans tous les documents d'époque que nous avons consultés, il n'y en a jamais plus de quinze d'énumérés. Voir idem, 1926: 17 et 1927: 27.
} 
lution des effectifs, la fondation de syndicats additionnels en 1926 et 1927, celle du syndicat des relieurs (Imprimerie Beauchemin) en particulier, a représenté une majoration notable du nombre de membres des syndicats de l'imprimerie de toute la province. Aux dires de leur porte-parole, les effectifs auraient quadruplé en 1926 pour atteindre plus de 400 membres $^{36}$. Jusqu'en 1925, l'ensemble du mouvement syndical catholique de l'imprimerie ne comptait en tout et pour tout qu' une centaine d'adhérents répartis dans trois villes: Montréal, Québec et Hull. Malheureusement, nous devons préciser que ces chiffres ne sont pas exempts d'exagération. En fait, nos propres calculs réalisés à partir de renseignements sur la valeur des assurances-décès détenues par tous les membres des syndicats concernés ${ }^{37}$ et des extrapolations à partir de la comparaison entre les effectifs déclarés et les effectifs réels du Syndicat catholique des relieurs de Montréal ${ }^{38}$ nous amènent à estimer à 250 environ le nombre de membres de tous les syndicats catholiques d'imprimeurs réunis. En eux-mêmes ces chiffres donnent une bonne idée de l'ampleur du mouvement syndical catholique dans l'imprimerie; ils montrent également les limites du pouvoir clérical en matière de promotion du syndicalisme confessionnel dans cette industrie. Mais la comparaison avec les effectifs unionistes est encore plus éloquente.

Tout en faisant remarquer que les internationaux ont également tendance à exagérer le nombre de leurs adhérents, il reste tout de même que la mise en parallèle des effectifs des Conseils des métiers alliés de l'imprimerie de Montréal et de Québec, présentés précédemment, et ceux des syndicats catholiques d'imprimeurs révèle que ces derniers n'ont représenté que 5.5\% des effectifs internationaux en 1921 et $7 \%$ en 1925 si l'on se fie aux chiffres fournis par les syndicats catholiques eux-mêmes. Avec l'augmentation des effectifs déclarés de 1926, ils auraient réussi à regrouper l'équivalent du quart des effectifs des unions internationales de 1929. Vraisemblablement, si nos estimations sont justes, ils n'ont pas dépassé plus de $15 \%$ de ces effectifs. Il y a toutefois plus impressionnant encore. Alors que les unions internationales de Montréal comptent en moyenne 196 à 260 membres chacune, et celles de Québec environ 62, les syndicats catholiques n'en comptent que 31 en $1927 ! \ldots$

36 CTCC, Procès-verbal du Congrès, 18 sept. 1926, 66. Le nombre déclaré de membres demeurera sensiblement le même jusqu'en 1936.

37 Les dirigeants des syndicats déclarent que leurs membres détenaient $225000 \$$ d'assurances-décès en 1928 dont les primes variaient entre $500 \$$ et $5000 \$$. Nous avons arbitrairement fixé la prime moyenne à $1000 \$$ pour ainsi arriver à un total de 225 membres assurés. Les renseignements relatifs aux assurances se trouvent dans Programme-Souvenir..., 1928: 31.

38 Dès sa fondation en 1926, on déclare que ce syndicat compte cent membres. Or, à aucun moment entre 1926 et 1929 il n'a regroupé plus de 64 adhérents. Nous avons donc retenu cette proportion de $64 \%$ entre les effectifs déclarés et les effectifs réels pour faire notre estimation de ceux de l'ensemble des syndicats catholiques d'imprimeurs. Voir idem, 1926: 5 et Minutes..., 1926 à 1929 inclusivement. 
Que conclure sinon que le syndicalisme catholique d'imprimeurs n'a réussi durant cette période de 1921 à 1929 à se consolider qu'en tant que mouvement syndical extrêmement minoritaire. Même le recours aux bonnes grâces du clergé n'a pas réussi à en faire un concurrent vraiment sérieux de l'unionisme international. De plus, les limites de cette stratégie de développement imposaient un changement d'orientation sans quoi on s'exposait à une stagnation à long terme ou à une régression rapide. La nécessité de ce changement sera mise encore plus en évidence par la crise économique de 1929 et ses effets sur le mouvement syndical catholique.

\section{Les réactions à la crise de 1929}

Les syndicats affiliés à la Fédération catholique des métiers de l'imprimerie du Canada ont adopté des attitudes très diverses à l'égard des problèmes engendrés par la crise. Pour le syndicat catholique des imprimeurs du journal Le Droit, l'impossibilité de renouveler son contrat de 1931 tout en gardant des salaires décents ${ }^{39}$ va le placer dans une impasse. Comme il était bien malvenu d'entreprendre des actions contre ceux qui avaient en partie assuré sa création, il décida tout simplement de se dissoudre et d'attendre des jours meilleurs pour reprendre ses activités ${ }^{40}$. Ces temps plus cléments se présenteront effectivement... en 1939, soit sept ans plus tard. Il semble donc que les circonstances qui ont entouré la formation de ce syndicat, en particulier l'intervention des Oblats, aient énormément hypothéqué les capacités de revendication de ces syndiqués.

Pour sa part, le Syndicat catholique et national des imprimeurs de Chicoutimi (SCNIC) réagira tout différemment à la situation de crise. Il n'y a pas de doute que le monopole de représentation qu'il détient dans la région a beaucoup influencé ses réactions. Il a bien sûr fait preuve de modération tant dans ses demandes que dans ses réponses aux offres patronales. Par exemple, ses membres à l'emploi du Progrès du Saguenay ont accepté coup sur coup durant l'automne 1930 la réduction de leur semaine de travail de 48 à 42 heures, puis à 40 heures, avec une réduction proportionnelle de leurs salaires. En novembre, il y a eu une nouvelle réduction des heures de travail accompagnée de coupures dans le personnel du journal ${ }^{41}$. En mars 1931, l'employeur récidive en demandant une diminution de $10 \%$ du travail et la suppression du temps supplémentaire $^{42}$. Le 14 mai 1932, la publication même du journal doit être suspendue pour ne reprendre que le 8 juillet. $\grave{A}$ cette occasion, le

\footnotetext{
39 Programme-Souvenir..., (1932): 21.

40 Album-Souvenir..., 54.

${ }_{41}$ Livre des procès-verbaux du Syndicat catholique et national des imprimeurs de Chicoutimi (désormais Livre...), sept. 1930: 27; 5 nov. 1930: 133; 31 nov. 1930: 134.

42 Idem, 11 avril 1931, 145.
} 
Progrès cesse d'être un quotidien et devient un hebdomadaire ${ }^{43}$. Il faut croire que c'est le prix qu'il a fallu payer pour relancer le journal. Malheureusement, ce n'est pas le seul. On note en effet dans les procèsverbaux des assemblées du SCNIC que les salaires des employés syndiqués du Progrès n'ont pas été versés depuis la reprise de la publication du journal jusqu'au printemps de 1933, c'est-à-dire pendant sept à huit mois ${ }^{44}$ ! Mécontents de cet état de choses, les syndiqués décident de recourir à la grève pour obtenir gain de cause. Déclenchée le 2 mars, elle durera vraisemblablement jusqu'au 12 juillet suivant ${ }^{45}$. Malgré sa durée, ou à cause d'elle, elle s'est finalement soldée par une défaite puisque la parution du journal n'a pu être empêchée et que le conflit s'est éternisé bien au-delà de ce que les ressources syndicales pouvaient absorber ${ }^{46}$. Cependant, plus que la défaite, ce qu'il faut retenir de cette grève, c'est le précédent qu'elle représente dans les annales du syndicalisme catholique de l'imprimerie, en plus d'être la plus longue lutte menée par des affiliés de la FCMIC entre 1921 et 1945.

Les formes différentes que revêt la crise à Montréal nécessitent par ailleurs le recours à de nouveaux moyens pour remédier aux problèmes qui assaillent les travailleurs de l'imprimerie. Entre la dissolution et l'affrontement, les syndicats catholiques d'imprimeurs de la ville se fraient donc péniblement un chemin. A cet endroit, la concurrence sur les salaires et la réduction des heures de travail prennent de telles proportions que beaucoup de travailleurs-imprimeurs n'ont que le choix de «partir à leur compte». Léonce Girard, l'agent d'affaires de la FCMIC, définit très clairement, d'un point de vue syndical, la cause et la nature de ce phénomène.

Sans se demander jusqu'à quel point l'ouvrier pouvait être responsable du malaise [c'est-à-dire de la crise], bon nombre de patrons, malgré l'expérience des autres métiers, se hâtèrent de chercher un remède dans la diminution des salaires et par une concurrence déloyale sur les gages des employés, forcèrent les autres imprimeurs à accepter leur solution inintelligente.

La généralisation de ce procédé eut pour effet de plonger définitivement l'industrie dans le marasme. Privés de salaires raisonnables, les ouvriers cherchèrent à accroître leurs revenus en ouvrant à domicile un petit atelier. Ils augmentèrent ainsi le nombre des

\footnotetext{
43 R. Lapointe, Histoire de l'imprimerie du Saguenay, 1879-1969 (Chicoutimi, Société Historique du Saguenay, \#22, 1969), 55 et 58

44 Livre..., 20 fév. 1933: 153-155 et 3 mars 1933: 163-168.

$45 \mathrm{Au} 28$ juin, la grève dure toujours, mais la mention de la signature d'un nouveau contrat le 12 juillet nous laisse croire qu'elle prit fin à ce moment. Voir idem, 2 mars 1933: 161; 28 juin 1933: $11-12$ et 12 juillet 1933: 20 .

46 C'est la conclusion qui s'impose quand on regarde la fréquence des assemblées syndicales du SCNIC. Il y en a eu 12 en mars, une en avril et aucune en mai. Il faut dire qu'il n'existait pas de fond de grève pour appuyer les grévistes à cette époque; ce qui rend la lutte beaucoup plus ardue.
} 
boutiques de fond de cours et de chambre à coucher qui sont devenues une véritable plaie dans notre ville. ${ }^{47}$

En offrant eux-mêmes des produits de moindre qualité, peut-être, mais à des prix très inférieurs, les travailleurs-imprimeurs des boutiques de fond de cours accéléraient encore la chute des prix et celle des salaires qui la suit inévitablement. Au lieu d'améliorer la situation, ils participaient à son aggravation. En plus, leur action était perçue par certains, dont L. Girard, comme étant la cause de «l'avilissement du métier» ${ }^{48}$. Les syndicats catholiques de l'imprimerie montréalaise essaieront de remédier à la situation à l'aide des moyens traditionnels, telle la conclusion de contrat de travail avec des maîtres-imprimeurs possédant un véritable esprit social ${ }^{49}$, la promotion de leur étiquette syndicale ${ }^{50}$, la demande de protection aux autorités religieuses et civiles relativement à la concurrence étrangère ${ }^{51}$, etc. Mais aucun de ces remèdes n'eut de résultat notable. C'est à la lumière de cet échec, du resserrement de ce cercle vicieux autour des travailleurs de l'imprimerie que l'on comprend l'importance d'une stabilisation des salaires. C'est dans ce but que la FCMIC fera preuve d'innovation en revendiquant l'instauration d'un système d'extension juridique des contrats collectifs de travail.

\section{L'extension juridique des contrats de travail}

L'extension juridique consiste à étendre une entente relative aux conditions de travail, intervenue entre un employeur ou un groupe d'employeurs et un syndicat ou un groupe de syndicats, à toutes les entreprises d'un territoire donné et d'une industrie donnée au moyen d'un décret gouvernemental pourvu que ces conditions aient «une signification et une importance prépondérantes ${ }^{52}$ dans l'industrie concernée. C'est, en fait, une sorte de convention collective minimale dont une partie des clauses s'applique à tous les travailleurs du secteur industriel visé, qu'ils soient syndiqués ou non. L'idée d'une législation de ce genre a d'abord été défendue par l'abbé Boileau, aumônier des Syndicats catholiques de Montréal, en $1929^{53}$. Mais c'est la Fédération

\footnotetext{
47 La Vie syndicale, nov. 1933: 1.

48 Ibidem.

49 Idem, août 1933: 5. déc. 1931: 9 .

Une grande campagne à cet effet fut lancée en 1931 par la FCMIC et la CTCC. Idem,

51 Idem, août 1933: 5.

52 S.Q., 24 Geo. V, ch. 56, art. 2. Pour plus d'informations sur cette loi voir J.-R. Cardin, L'influence du syndicalisme national catholique sur le droit syndical québécois (thèse M.A., U. de M., 1948), 56 à 87 principalement. Aussi, C. St-Pierre, Le développement de la société québécoise, saisie à travers l'analyse des orientations et des pratiques du syndicalisme catholique et des unions internationales, la définition des idéologies dominantes et la mise à jour des contradictions fondamentales (1929-1940) (thèse de Doctorat de $3^{\mathrm{e}}$ cycle, EPHE, VI ${ }^{\mathrm{e}}$ section, Paris, juin 1973), 495 à 515.

${ }_{53}$ Voir J. Rouillard, Histoire de la CSN..., 138 et M.-L. Beaulieu, «Législation du travail, La Loi de la convention collective (S.R.Q., 1941, ch. 13)», dans La Revue du Barreau, 14, \#8 (oct. 1954): 385 .
} 
catholique des métiers de l'imprimerie qui, la première, en a fait une proposition en bonne et due forme au congrès de la CTCC de $1931^{54}$. Cette proposition réclamait de la CTCC qu'elle lance une campagne d'éducation sur l'extension juridique afin de familiariser tant les patrons, les syndiqués que les législateurs avec ce genre de mesure. Malgré son caractère modéré et l'appui qu'elle a reçu du Comité des résolutions du congrès, l'adoption de la proposition a été différée à la suite de l'intervention de l'abbé Maxime Fortin, aumônier général de la $\mathrm{CTCC}^{55}$. Celui-ci et quelques autres dirigeants de la centrale estimaient que la mesure proposée était trop radicale ${ }^{56}$. L'année suivante, la proposition est représentée et à nouveau mise sur les tablettes ${ }^{57}$.

L'adoption de positions aussi contradictoires par deux aumôniers de syndicats catholiques à propos de l'applicabilité de l'extension juridique au Canada s'explique par les problèmes que son utilisation éventuelle suscite au plan de la doctrine sociale de l'Église. Les attitudes divergentes de l'abbé Boileau et de l'abbé Fortin sont une des manifestations des tiraillements qui ont cours à l'intérieur de l'Église catholique de l'époque entre les tenants d'un corporatisme d'État quelque peu fascisant et ceux d'un corporatisme social ${ }^{58}$. La différence essentielle entre ces deux tendances est que la première préconise une centralisation absolue des pouvoirs aux mains de l'appareil étatique dans tous les domaines de la vie sociale, y compris celui des relations de travail; tandis que le second mise plutôt sur la création d'organismes intermédiaires, civils, d'organisations regroupant les employeurs et les travailleurs d'un même secteur professionnel par exemple, pour contrebalancer le pouvoir d'État et parvenir à une société corporative conforme aux principes chrétiens. On voit donc que la proposition de la Fédération catholique de l'imprimerie se retrouve au coeur d'une bataille doctrinale d'envergure. Ce qui doit être souligné ici, c'est que tant que cette question doctrinale ne sera pas réglée d'une façon ou d'une autre, la promotion de l'extension juridique des contrats de travail devra attendre. Deux événements vont contribuer à ce que le problème soulevé par la demande des imprimeurs catholiques soit finalement résolu: la publication de l'encyclique Quadragesimo Anno par le pape Pie XI en 1931 et l'accentuation de la crise économique dont le creux est atteint en $1933^{59}$.

L'encyclique de 1931 représente en quelque sorte la victoire du corporatisme social sur le catholicisme dit de droite. Il constitue donc

54 CTCC, Procès-verbal du Congrès, 1931, rés. \#31.

55 La Vie syndicale, sept. 1931: 7.

56 J.-R. Cardin, op. cit., 61-62 et M.-L. Beaulieu, loc. cit., 385.

57 CTCC, Procès-verbal du Congrès, 1932, rés. \#9.

58 Voir l'article extrêmement intéressant de G.-R. Laliberté, «Dix-huit ans de corporatisme militant, l'École sociale populaire de Montréal, 1933-1950», dans Recherches sociographiques, XXI, \#2, (1980): 55 à 96 dont nous reprenons une partie de l'argumentation pour expliquer les positions cléricales face à l'extension juridique.

59 Idem: 68. 
un outil fondamental de réflexion et d'étude pour les tenants québécois de la doctrine sociale de l'Église, qu'ils soient clercs ou laïcs. De fait, les exposés présentés aux séances des Semaines sociales du Canada de 1931 et 1932 vont puiser très abondamment dans cet encyclique pour mieux comprendre le rôle de l'État dans la société et pour mieux définir ce que devrait être l'Ordre social chrétien ${ }^{60}$. Mais on en est encore aux considérations générales et, en conséquence, la détermination d'une ligne d'action concrète reste encore à élaborer. L'approfondissement de la crise économique met toutefois le clergé face à face avec les limites de sa lutte contre la misère via le syndicalisme. Comme le dit G.-R. Laliberté, aux yeux de celui-ci «le syndicalisme en tant que promotion de la condition ouvrière ne suffit plus; il faut lui associer un programme social complet qui porte le débat sur la totalité de l'organisation sociétale» ${ }^{61}$. Autrement dit, la lutte doit s'étendre au terrain politique. Le résultat de cette prise de conscience est la publication à l'automne de 1933 du Programme de restauration sociale dont le but est de faire la promotion d'une organisation corporative adaptée à la société québécoise et dans laquelle l'extension juridique figure en bonne place $^{62}$. Cette période de réflexion intensive étalée sur deux ans correspond exactement avec celle de la mise en dépôt de la proposition de la FCMIC; ce n'est pas qu'un hasard.

On peut également avancer que c'est la prise de conscience des limites de l'action syndicale en temps de crise économique qui conditionne l'attitude des membres de la FCMIC et celle des travailleurs de l'imprimerie appartenant aux unions internationales à l'égard de l'extension juridique. À cette différence près que la FCMIC bénéficie d'une longueur d'avance dans la recherche de moyens susceptibles de pallier à une trop grande faiblesse des organisations syndicales parce que son pouvoir de pression dans l'industrie laissait déjà à désirer même avant l'arrivée de la crise. Ainsi, les internationaux ne s'intéresseront à l'extension juridique qu'à partir du moment où la CTCC demandera un projet de loi en ce sens au gouvernement, c'est-à-dire à l'automne de $1933^{63}$. De plus, il est bien évident que cette prise de conscience et que les attitudes qu'elle a déterminées s'exprimeront, de part et d'autre, à l'intérieur des paramètres des doctrines syndicales propres au syndicalisme catholique et à l'unionisme international. Chez les catholiques, l'extension juridique est conçue et présentée comme un moyen de

\footnotetext{
60 Voir Semaines sociales du Canada, L'État, Compte rendu des Cours et Conférences, $10^{\mathrm{e}}$ session (Mtl., Secrétariat des Semaines Sociales du Canada, ESP, 1931) et L'Ordre social Chrétien, Compte rendu des Cours et Conférences, $11^{\mathrm{e}}$ session (Mtl., Secrétariat des Semaines Sociales du Canada, ESP, 1932), 393 p.

61 G.-R. Laliberté, loc. cit., 69.

62 Au sujet de ce programme, voir J. Cousineau, s.j., L'Église d'ici et le social, 1940-1960 (Mtl., Bellarmin, 1982), 132 à 141 ainsi que M. Pelletier et Y. Vaillancourt, Les politiques sociales et les travailleurs, Cahier II: Les années '30 (Mtl., 1975): 127 à 136.

63 Le Monde Ouvrier, 9 sept. 1933: 1 et 4; 6 janv. 1934: 1 .
} 
redonner toute sa valeur à la Loi des syndicats professionnels de 1924 et même d'en combler les lacunes ${ }^{64}$. C'est ce qui apparaît clairement dans un article paru dans l'organe des syndicats catholiques nationaux.

Lorsque le Gouvernement Provincial, sur la demande des Syndicats Catholiques, passa la loi des syndicats professionnels, légalisant la convention collective, il concéda aux travailleurs syndiqués des privilèges très appréciables: celui de pouvoir réclamer devant les tribunaux lorsque le contrat de travail est violé et celui de régler par des moyens légaux, plutôt que par la grève, les difficultés entre patrons et ouvriers.

Ces privilèges, très grands en théorie, n'en restent pas moins minimes en pratique. Il est excellent d'avoir le droit de signer des contrats collectifs de travail dont le but est de supprimer les conflits entre patrons et ouvriers et de prévenir les grèves. Mais comment faire signer ces contrats collectifs? Les patrons qui ont véritablement le sens social et qui sont prêts à payer des salaires raisonnables à leurs travailleurs lorsque leurs concurrents paient des salaires de famine se font de plus en plus rares. Reste aux ouvriers un moyen de signer des contrats de travail, c'est de les imposer par la force, à la suite d'une grève. Et alors, le but de la convention collective, qui est de prévenir la grève et le désaccord, se trouve manqué. Les conventions collectives imposées porteront toujours en elles-mêmes le discrédit de la lutte et ne sauront durer que le temps où un groupe pourra les imposer. ${ }^{65}$

Ce qui apparaît aussi dans cette citation, c'est que les syndicats catholiques sont bien conscients, maintenant, du rapport de force inévitable et même nécessaire qui existe entre patrons et ouvriers. La question à résoudre est de savoir par quels moyens ce rapport peut être orienté vers la promotion des intérêts des travailleurs. Pour ces syndicats, la réponse se trouve dans le recours au pouvoir législatif de la province; voilà qui explique la nature de la proposition de la FCMIC de 1931.

Par ailleurs, cette position contraste radicalement avec celle des unions internationales. On peut en juger par ces propos du secrétairetrésorier de l'Union typographique Jacques-Cartier (local \#145 de l'UTI): «en dépit des bienfaits que cette loi [de l'extension juridique] doit apporter (...), nous persistons à croire et à dire que ce n'est que par la force économique de leurs organisations que les ouvriers parviendront au bien-être qui leur est dû.. ${ }^{66}$ Comme on peut en juger par ces propos, avant son adoption et, ultérieurement, durant une courte période, les unionistes ne s'opposeront pas à la loi dans son principe; compte tenu de l'affaiblissement de la force économique de leurs unions durant la crise, compte tenu également des effets pratiques que l'on

64 Sur cette loi voir J.-R. Cardin, op. cit., 35 à 53.

65 La Vie syndicale, sept. 1933: 1.

66 Le Monde Ouvrier, sept. 1934: 4. 
peut escompter de cette loi au plan de la stabilisation des salaires, cette mesure apparaît comme un palliatif somme toute acceptable. À un point tel que certains en feront la promotion ${ }^{67}$. C'est pour ces raisons que certaines unions internationales, les unions d'imprimeurs y compris, participeront au régime d'extension juridique malgré la dénonciation qu'elles feront d'autres aspects de la loi.

Dans un premier temps, elles n'ont pas manqué en effet de formuler de sérieuses réserves à son égard, s'y opposant dans la mesure où elles refusaient d'en faire une panacée et dans la mesure où la loi pouvait devenir un outil de promotion du syndicalisme catholique ou un moyen détourné de rendre obligatoire l'incorporation des associations de travailleurs ${ }^{68}$. Si cette dernière éventualité était devenue un état de fait, elle aurait signifié que les syndicats catholiques auraient joui du droit exclusif d'influencer l'ensemble des conditions de travail prévalant dans une industrie donnée ${ }^{69}$. Dans le cas de l'imprimerie par exemple, cela aurait conféré une importance démesurée aux 380 syndiqués catholiques de Montréal par rapport aux quelque 1059 unionistes de cette même ville en $1934^{70}$. Heureusement pour les internationaux, suite à la manifestation de leur opposition à une telle mesure, les dispositions relatives à l'incorporation des associations de travailleurs n'apparaitront pas dans le texte de loi adopté le 20 avril $1934^{71}$.

Par la suite, à mesure que la diffusion du Programme de restauration sociale s'élargissait, et avec elle l'idée que l'extension juridique constituait la première réalisation en vue de l'instauration du corporatisme, les internationaux ont dénoncé avec toute l'énergie dont ils disposaient cette idéologie et ce projet de société qui, pour eux, menaient directement au fascisme ${ }^{72}$.

Le rejet du corporatisme par les internationaux et leur réussite dans la lutte contre l'incorporation obligatoire n'annihilent pas pour autant les dispositions susceptibles de conférer aux syndicats catholiques les avantages et privilèges qu'ils comptent bien retirer de l'extension juridique. En fait, le processus même d'application de cette législation fait en sorte que les syndicats catholiques acquièrent un pouvoir sans commune mesure avec leur force réelle. C'est du moins ce que laisse croire l'exemple des syndicats catholiques de l'imprimerie.

\footnotetext{
67 G. Francq, rédacteur du Monde Ouvrier, fera campagne en faveur de cette loi. En plus des considérations toutes syndicales, ses liens avec le parti libéral sont sûrement pour beaucoup dans l'adoption de cette position. Voir Le Monde Ouvrier, 10 février 1934, 1 entre autres.

68 Idem, 6 janv. 1934: 1; 24 fév. 1934: 1.

69 Idem, 17 fév. 1934: 1

70 Ces chiffres sont tirés de Ministère du Travail, L'Organisation ouvrière au Canada, (Ottawa, 1934), 178.

71 Voir le texte intégral de cette législation dans Le Monde Ouvrier, 5 mai 1934: 4.

72 Les membres du Parti communiste du Canada partageaient la même position à propos de la loi de 1934. Voir B. Gauvin, Les communistes et la question nationale au Québec (Mtl., Les Presses de l'unité, 1981), 89.
} 
D'abord, le mode de représentation au Comité paritaire chargé de voir à l'application et au respect des conventions collectives extensionnées se fait sur la base d'un nombre équivalent de représentants par organisation syndicale existante et non en fonction du nombre de membres représentés par chacune d'elle ${ }^{73}$. Les effectifs respectifs des syndicats catholiques et des unions internationales étant ce qu'ils sont, la faveur consentie par la loi aux premiers est évidente. À cet égard, il est pertinent de souligner que ce sont d'abord et avant tout les syndicats catholiques de l'imprimerie montréalaise, les plus exposés à la concurrence des internationaux, qui se sont faits les promoteurs de l'extension juridique et que, d'autre part, ce sont eux qui éprouveront le plus de difficultés à s'en prévaloir.

En effet, le Syndicat catholique des imprimeurs-relieurs de Québec sera le premier à bénéficier de l'extension juridique en obtenant un premier décret, le 11 juillet 1934, dans lequel les unions \#302 (UTI) et \#152 (Union internationale des pressiers d'imprimerie) figurent parmi la partie syndicale ${ }^{74}$. L'obtention d'un décret ne semble pas poser de problème au syndicat catholique; lors de son renouvellement, il réussira même à en conclure un nouveau sans la participation des unions internationales ${ }^{75}$, ce qui laisse entendre qu'elles ne sont pas prépondérantes à cet endroit. Pour sa part, le Syndicat catholique et national des imprimeurs de Chicoutimi obtiendra sa première convention collective extensionnée, applicable à toute la région du Saguenay-Lac St-Jean, dès le 24 août $1935^{76}$. Pendant longtemps, l'obtention de telles conventions par ce syndicat ne sera tout au plus qu'une question de formalité puisqu'il était le seul à représenter les travailleurs-imprimeurs de la région et que de toutes façons, il ne concluait pas de contrat particulier avec chacune des entreprises sous sa juridiction mais plutôt un contrat unique s'appliquant à leur ensemble $\mathrm{e}^{\dagger 7}$. Enfin, la signature de la première convention collective extensionnée couvrant la région de Montréal ne se fera que le 15 février $1936^{78}$, en grande partie à cause du peu d'empressement des unions internationales ${ }^{99}$. Le privilège conféré aux syndicats catholiques par le mode de représentation au Comité paritaire explique selon nous l'hésitation des internationaux.

À un autre niveau, l'inclusion dans les dispositions obligatoires des décrets de clauses relatives à la durée et aux règles de l'apprentissage ${ }^{80}$ constitue nettement la négation de certaines préroga-

\footnotetext{
73 Les unions internationales contesteront ce mode de représentation seulement le 15 septembre 1960 dans un mémoire présenté à R. Hamel, ministre du Travail de la province.

74 Gazette officielle du Québec, 29 sept. 1934, 4228.

75 FCMIC, Procès-verbal du Congrès, 9 oct. 1937, 89.

76 Gazette officielle du Québec, 24 août 1935, 3560.

77 L'Imprimeur, 5 sept. 1951, 3.

78 Minutes..., 19 fév. 1936: 8

79 Idem, 18 juillet 1934: 132-133; 17 juillet 1935: 173-174 et 18 déc. 1935: 183.

80 J.-R. Cardin, op. cit., 76.
} 
tives détenues par les internationaux dans ce domaine. L'UTI par exemple considère depuis toujours que les questions qui concernent l'apprentissage du métier sont du ressort exclusif des syndicats et qu'en aucun cas elles ne sont négociables ou arbitrables ${ }^{81}$. C'est pourquoi elle possède sa propre école de métier, au même titre que l'Union internationale des pressiers. Évidemment, la FCMIC ne peut se payer un tel luxe. L'extension juridique s'avère donc un moyen peu coûteux de pallier à son manque de ressources. Une étape supplémentaire sera d'ailleurs franchie en ce domaine avec la création de l'École des Arts graphiques par le gouvernement provincial en août $1942^{82}$.

Ces deux illustrations des avantages consentis aux syndicats catholiques de l'imprimerie par l'extension juridique nous amènent à formuler l'hypothèse suivante, à savoir que dans le but de s'assurer l'influence que leurs seules ressources ne suffisaient pas à établir, les syndicats catholiques d'imprimeurs ont eu recours à l'Etat provincial comme source d'appui. En requérant les pouvoirs législatifs de cet État, ils ont réussi à obtenir un appui qui contrebalançait la force numérique des organisations internationales. Le recours à la collaboration de l'État, peu importe la forme qu'il a pu revêtir, s'avérait indispensable, à notre avis, à la survie et au développement des syndicats catholiques de l'imprimerie. Comment une fédération catholique ne comptant que 484 membres en $1936^{83}$ et dont la juridiction se limitait à trois régions du Québec pouvait-elle rivaliser avec succès avec les trois unions internationales qui lui faisaient directement concurrence? D'autant plus que ces unions, celle des typographes, celle des pressiers et celle des relieurs, recrutaient leurs membres dans toute l'industrie de l'imprimerie nordaméricaine et regroupaient des centaines de fois plus de membres que la fédération catholique ${ }^{84}$.

Selon nous, la FCMIC a préconisé très tôt la mise en vigueur de l'extension juridique entre autres choses parce que, en tant qu'organisation syndicale, elle y avait intérêt. Il ne s'agit pas de prétendre que cette législation n'avait que des fins partisanes. La fixation par voie législative de conditions minimum de travail durant une période de crise économique comme celle des années 1930 représente indéniablement un atout important pour freiner la détérioration de la condition ouvrière, même si, par ailleurs, il est difficile d'en mesurer les effets concrets dans le cas de l'imprimerie puisque son application dans la majeure

\footnotetext{
81 Cette conception des prérogatives syndicales en matière d'apprentissage sera en vigueur tant dans les années 1930 que 1940. Des conflits de travail éclateront spécifiquement à ce propos d'ailleurs. Voir H.A. Logan, op. cit., 114 à 116.

82 L'Imprimeur, mai-juin 1950, 14

83 FCMIC, Procès-verbal du Congrès, 11 sept. 1937, 66.

84 L'UTI, à elle seule, compte 4200 adhérents au Canada et 74300 en Amérique du Nord. Voir H.A. Logan, op . cit., 617.
} 
partie de l'industrie coïncide presque année pour année avec la reprise des affaires, c'est-à-dire en $1936^{85}$.

Nous croyons également que les internationaux ont boudé pendant quelques années ce système de règlementation des conditions de travail en partie parce que cette participation signifiait une diminution relative de leur influence dans la détermination des conditions de l'industrie, à l'avantage des syndicats catholiques. Il est significatif à cet égard que ce ne soit qu'à partir de la reprise économique dans l'industrie et, en conséquence, à partir du raffermissement de ce qu'ils appellent leur force économique qu'ils consentiront à participer au processus d'extension d'une convention collective pour la région de Montréal. À nos yeux, cette attitude indique que pour eux, cette mesure législative n'est qu'un palliatif aux problèmes des travailleurs et que, même dans le domaine de la rivalité inter-syndicale, c'est la force conférée par l'adhésion du plus grand nombre possible de travailleurs qui demeure le facteur fondamental et déterminant de toute stratégie.

Les syndicats catholiques sont aussi conscients de cette réalité mais à cause de l'opinion défavorable qu'ils se font de toute stratégie visant à construire un rapport de force direct entre patrons et ouvriers, surtout si elle implique l'utilisation de la grève, et à cause des limitations qu'impose leur situation, ils ont choisi la voie législative. Si en période de crise économique un tel choix s'avère profitable, en-dehors de ces temps difficiles on peut douter de ses capacités à améliorer les conditions de vie et de travail des ouvriers. Il faut tout de même reconnaître qu'en utilisant cette voie, ils ont su profiter d'une certaine audience auprès du gouvernement provincial. D'abord, les préoccupations électoralistes de Taschereau ont sûrement été mises à contribution ${ }^{86}$. De plus, l'opportunité qui lui était offerte de favoriser un tant soit peu les concurrents de l'UTI, avec laquelle il avait eu maille à partir durant le Mouvement des 44 heures, ne devait sûrement pas trop lui déplaire ${ }^{87}$. Ensuite, il faut aussi compter l'influence cléricale au nombre des éléments favorables aux imprimeurs catholiques. Finalement, durant les années 1930, la CTCC avait d'ores et déjà acquis le statut d'institution authentiquement canadienne-française; cela lui confère sans doute, ainsi qu'à ses corps affiliés, un atout dans ses rapports avec le gouvernement provincial.

\footnotetext{
85 L'article de R. Gaudreau, «Le comportement des salaires dans l'industrie de l'imprimerie de Montréal et du district, 1940-1949», dans Bulletin des Relations industrielles (juillet 1950): 95 à 97 n'arrive pas selon nous à montrer les retombées positives de l'extension juridique à ce niveau .

86 M.-L. Beaulieu, loc. cit., 385.

87 Voir à ce sujet J. Rouillard, Les Syndicats..., 299 à 305 et R. Rumilly, Histoire de la province de Québec, XXVI: 45 à 48.
} 


\section{Le temps de guerre}

Pendant les années de guerre, la rivalité entre le mouvement syndical catholique et les unions internationales atteint son paroxisme. Au même moment, la situation est telle que J. Cousineau, qui fut aumônier de Conseils centraux et de Fédérations professionnelles catholiques durant cette époque, n'hésite pas à parler d'anarchie dans les relations patronales-ouvrières au Québec ${ }^{88}$. Pendant cette période mouvementée, c'est toutefois la collaboration inter-syndicale qui prévaut dans l'industrie de l'imprimerie.

Pourtant, la formation de la Fédération des unions internationales des métiers de l'imprimerie (FUIMI) en juillet 1941 n'est pas de nature à susciter un rapprochement des syndicats présents dans ce secteur ${ }^{89}$. Son objectif principal étant justement de déborder le territoire traditionnellement occupé par les imprimeurs des unions internationales, c'està-dire Montréal, et d'étendre leur présence aux autres centres industriels de la province, les perspectives de confrontation avec les syndicats catholiques sont évidentes. Ceux-ci se proposent d'ailleurs de prendre les devants en lançant une grande campagne d'organisation dans le but de contrecarrer les visées des internationaux ${ }^{90}$. L'entrée en guerre du Canada et la promulgation du décret $8253^{91}$ par le gouvernement fédéral dans le but de limiter les augmentations salariales sont venues différer l'affrontement qui se dessinait et en provoquer un d'une tout autre nature.

Disons tout de suite que cette législation avait de quoi déplaire aux travailleurs catholiques de l'imprimerie. Certains d'entre eux venaient justement d'accepter en avril 1941 un contrat presque identique au précédent en espérant en obtenir un meilleur à la fin de l'année ${ }^{92}$. Ceux de l'Illustration Nouvelle avaient réussi à obtenir des augmentations de $40 \%, 45 \%$ et même $50 \%$ dans certains cas devant être versées entre le mois d'août 1941 et le 2 janvier $1942^{93}$. Or, les stipulations du décret fédéral qui avaient priorité entraient en vigueur dès le 15 novembre $1941^{94}$. Ce n'était évidemment pas du goût des syndiqués catholiques.

Les années à venir promettaient en plus des gains appréciables compte tenu de la pénurie de main-d'oeuvre qualifiée dûe à la guerre et

$88 \quad$ J. Cousineau, op. cit., 144.

89 La FUIMI fut fondée le 15 juillet. Elle comprenait, au 26 de ce mois, l'Union des typographes de Montréal \#176, l'Union internationale des pressiers et assistants \#52, l'Union internationale des relieurs \#91, l'Union internationale des clicheurs et stéréotypeurs \#33 et l'Union typographique Jacques-Cartier \# 145. Toutes les informations relatives à la FUIMI, sauf indication contraire, sont tirées de La Presse, 26 juillet 1941, 44.

90 FCMIC, Procès-verbal du Congrès, 12-13 sept. 1941: 477.

91 Le décret fut voté le 24 oct. 1941. L'Imprimeur, \#9, fév. 1942, 1.

92 Idem, \#9, janv. 1943, 1.

93 Minutes..., 20 août 1941: 190.

94 L'Imprimeur, \#9, fév. 1942, 1. 
à l'accélération du rythme de développement technique et commercial de l'industrie. Il manquait en effet 500 hommes de métier en 1945 pour satisfaire aux besoins de l'industrie ${ }^{95}$. Pour illustrer le développement impressionnant des besoins en main-d'oeuvre, il suffit de mentionner que le pourcentage moyen d'accroissement des heures rémunérées à taux régulier pour les années 1938 à 1947 était de 40.4\%, celui des heures rémunérées à taux et demi de $106.8 \%$, et enfin que celui des heures rémunérées à taux double était de $187.6 \%^{96}$.

En conséquence, la Fédération catholique de l'imprimerie dénonce dès les premiers mois de sa mise en vigueur la teneur du décret fédéral, le qualifiant de «loi ridicule» et de «leurre pour les ouvriers» ${ }^{97}$. Comme on s'en doute, ces récriminations n'ont pas grand effet sur l'attitude du législateur. La FCMIC tente alors d'obtenir pour ses membres la maigre consolation d'un boni de vie chère semblable à celui que les employeurs sont enjoints de payer à tous les travailleurs assujettis à la législation de guerre. Mais les syndiqués de la Fédération ne sont pas couverts par cette législation spéciale parce que leur industrie n'est pas classée parmi les industries de guerre. C'est donc par son inclusion dans les conventions collectives qu'ils tentent de bénéficier du boni.

Certains travailleurs-imprimeurs non affiliés à la FCMIC avaient aussi adopté cette tactique et étaient parvenus très près du but.

Dans la lithographie [en effet], les employeurs et les ouvriers avaient conclu une entente au sujet du boni de vie chère. (...) Mais le jour où les intéressés demandèrent au gouvernement d'insérer cette entente dans la convention collective de l'industrie, le Conseil régional du travail leur déclara que, suivant le décret 8253 , le boni ne pouvait s'appliquer, de cette façon, à la collectivité. ${ }^{98}$

Ce genre de politique a soulevé un tel tollé de protestation qu'un amendement au décret fut adopté permettant ainsi aux clauses relatives au boni de vie chère d'être insérées dans une convention collective de travail et d'acquérir un caractère obligatoire ${ }^{99}$.

En janvier 1943, malheureusement, les imprimeurs ne profitent pas encore du boni autorisé l'été précédent. La FCMIC et la FUIMI décident donc de collaborer à l'atteinte de cet objectif commun ${ }^{100}$. Pour appuyer la demande des fédérations, G.-A. Gagnon, alors président de la FCMIC, soulève le cas des travailleuses des ateliers de reliure membres de son organisation qui n'ont pas reçu d'augmentation depuis sept

\footnotetext{
95 J. Villeneuve, L'apprentissage dans l'industrie de l'imprimerie de Montréal, 1936-1947, (thèse M.A., Rel. Ind., U. de M., 1949), 35-36.

96 Idem, 38.

97 L'Imprimeur, \#9, fév. 1942, 1.

98 Idem, \# 1, mai 1942, 1.

99 L'amendement date du 10 juillet 1942. Idem, \#9, janv. 1943, 1.

100 Sur cette collaboration voir idem, \#9, janv. 1943, 1 et \#12, avril 1943, 1.
} 
ans $^{101}$. Après avoir manifesté de bonnes dispositions à l'endroit de la requête des ouvriers imprimeurs, le Conseil régional du travail se ravise et refuse la demande des associations de travailleurs. Selon Gagnon, ce revirement d'attitude est dû à la réaction patronale envers les demandes syndicales. Selon lui, le Conseil aurait d'abord accordé délai par-dessus délai aux patrons pour leur permettre d'étudier la question. Puis, une enquête aurait été menée dont les résultats seraient les suivants: sur 536 maîtres-imprimeurs consultés, 215 ne seraient pas intéressés à la question, 128 n'auraient pas d'employé, 210 s'opposeraient à une hausse du bonus et 31 ne s'opposeraient pas à une légère hausse. Et Gagnon ajoute:

il est facile de constater que ce Conseil en est un du plus pur favoritisme patronal servant à engendrer le mépris de la classe ouvrière. (...) il n'ose prendre des décisions que lorsque des grèves se déclarent et qu'il se voit alors dans l'obligation d'agir. ${ }^{10}$

Il accuse le Conseil de se complaire dans sa «léthargie», sa «lenteur», son «favoritisme», son «indécision», d'être composé de «fonctionnaires-tumeurs qui provoquent la dégringolade du peu de législation sociales (sic)» qui existent, etc. ${ }^{103}$. Comme on le constate, l'idée que les dirigeants de la FCMIC se font du patronat et de certains organismes gouvernementaux a évolué énormément depuis le début des années 1930. La pratique syndicale a également changé: nous avons recensé au moins une menace de grève et six grèves effectives menées par des affiliés de la FCMIC entre mai 1938 et septembre $1944^{104}$. Bien que n'étant pas particulièrement spectaculaires et étant généralement de courte durée, ces conflits de travail dans une fédération imbue pendant longtemps de visées bonne-ententistes marquent, à n'en pas douter, une rupture de la pratique mais aussi de l'idéologie syndicale. On comprend mieux dès lors que Gagnon puisse tenir des propos aussi vigoureux à l'endroit du Conseil régional.

De concert avec la FUIMI, la FCMIC en appelle de la décision de l'instance régionale devant le Conseil national du travail en temps de guerre $^{105}$. Parallèlement à cette action commune, la Fédération catholique inscrit à l'ordre du jour de son congrès de septembre le recours à la grève générale dans toute l'industrie ${ }^{106}$. Les résultats ne se font pas

101 Idem, \#9, janv. 1943, 1. On peut s'interroger sérieusement sur les capacités et la volonté revendicatives de la FCMIC, ainsi que sur la place qu'y occupent ces travailleuses quand on sait qu'en 1936 la première proposition présentée pour et par des femmes dans les réunions du Syndicat catholique des relieurs de Montréal réclamait justement une amélioration des salaires. Six ans plus tard, rien n'avait bougé! Voir Minutes..., 20 janv. 1936: 5.

102 L'Imprimeur, \# 12, avril 1943, 1.

103 Ibidem.

104 Les sources ayant servi au recensement sont: Minutes..., 18 mai 1938: 98; 17 mai 1939: 124; 15 mai 1940: 154; 19 nov. 1941: 197; 18 nov. 1942: 220; 18 août 1943: 240. FCMIC, Procès-verbal du Congrès, sept. 1942: 559 et 18 sept. 1943: 639. L'Imprimeur, \# 5, sept. 1944.

105 L'Imprimeur, \#6, oct. 1943, 1.

106 FCMIC, Procès-verbal du Congrès, sept. 1943, 658. 
attendre. Avant la fin du mois, le Conseil national donne raison aux syndiqués et leur consent l'essentiel de leur demande ${ }^{107}$.

Une victoire de ce genre ne suffit malheureusement pas à assurer une progression équitable des conditions de travail. Selon une étude ultérieure, tant d'énergies et d'efforts dépensés ne sont pas parvenus à maintenir le rythme d'accroissement salarial établi avant $1941^{108}$. La perte de vitesse est d'autant plus grande qu'il faut pondérer le taux horaire moyen effectivement payé aux travailleurs en fonction de l'accroissement incroyable du nombre des heures supplémentaires travaillées dont nous avons fait mention plus haut. En dépit de cette note peu réjouissante, l'intervention fédérale aura au moins permis un certain rapprochement entre des organisations ouvrières jusque-là rivales à tous points de vue et l'adoption d'une attitude plus combative par la FCMIC.

$$
\text { ** }
$$

$\mathrm{Au}$ terme de cette exposé sommaire, nous pensons avoir montré que dans un premier temps, c'est la conjugaison de l'action cléricale en faveur d'un mouvement syndical catholique puissant et du mécontentement de certains imprimeurs à l'endroit de leurs unions internationales qui est le principe générateur du syndicalisme catholique dans l'imprimerie. En sa qualité de propriétaire d'ateliers d'imprimerie et de client important pour les entreprises de ce secteur, le clergé a joué un rôle non négligeable dans la promotion du syndicalisme confessionnel chez les imprimeurs. La création des syndicats catholiques de L'Action catholique, du journal Le Droit et de l'Imprimerie Beauchemin en témoigne amplement. Mais malgré leur importance, ces syndicats font figure d'exception tant du point de vue du type d'employeurs avec lesquels ils ont une entente que du point de vue de leur mode d'implantation dans chacune de ces entreprises. En effet, la majorité des syndiqués catholiques de l'imprimerie sont recrutés dans des entreprises canadiennes-françaises, certes, mais qui ne sont pas propriétés du clergé. Peut-être la force sociale indéniable que possède le clergé dans la société québécoise de l'époque a-t-elle joué un certain rôle dans l'adhésion de ces travailleurs à la FCMIC, mais c'est une influence bien difficile à mesurer.

Dans un second temps, le clergé éprouve certaines difficultés à endosser les mesures proposées par les imprimeurs catholiques pour contrer les effets désastreux de la crise économique. Les questions de doctrine étant les préoccupations premières des propagateurs de la doc-

\footnotetext{
107 La décision du Conseil national ne concerne en effet que les imprimeurs assujettis au décret de l'imprimerie de Montréal et travaillant dans la zone 1 seulement, c'est-à-dire sur l'île même et dans un rayon de 10 milles tout autour. Voir L'Imprimeur, \#6, oct. 1943, 1 et \#8, déc. $1943,4$.

R. Gaudreau, loc. cit., 96.
} 
trine sociale de l'Église, l'appui demandé par la FCMIC à la promotion de l'extension juridique des conventions collectives ne sera donc consenti que lorsque les éclaircissements doctrinaux jugés nécessaires auront été acquis. Une fois cet appui obtenu, la Fédération catholique doit faire face à l'attitude négative des unions internationales vis-à-vis de cette loi. Ces unions, en effet, prennent bien garde de ne laisser passer aucune mesure législative qui favoriserait indûment les syndicats catholiques ou qui représenterait une source additionnelle d'affaiblissement de leur propre force économique. Ce n'est que lorsqu'elles estimeront que leur prépondérance n'est plus menacée qu'elles participeront à l'extension juridique de contrats de travail.

Pendant la guerre, les conditions de l'industrie évoluent dans un sens très favorable aux travailleurs de l'imprimerie. Cela leur permet de se confronter à la fois aux politiques de temps de guerre du gouvernement fédéral et à une partie du patronat. C'est aussi une période propice à l'établissement d'une collaboration inter-syndicale et à l'adoption d'une attitude beaucoup plus combative par la FCMIC. 